\title{
Gated Photochromism of 1,2-Diarylethenes
}

\author{
Kühni Joël, and Peter Belser* \\ Department of Chemistry, University of Fribourg, rue du musée 9, 1700 Fribourg
}

Electronic supporting information

\section{Contents}

\section{Experimental}

2. Synthesis

3. Thermal stability

4. Fatigue resistance

5. Crystal structure

6. ${ }^{1} \mathrm{H}-\mathrm{NMR}$ and ${ }^{13} \mathrm{C}-\mathrm{NMR}$

\section{Experimental}

All products were characterized by ${ }^{1} \mathrm{H}-\mathrm{NMR}$ and ${ }^{13} \mathrm{C}-\mathrm{NMR}$, on a Bruker Advance DRX400 (400.13 MHz, for ${ }^{1} \mathrm{H}-\mathrm{NMR}$ and $100.62 \mathrm{MHz}$ for $\left.{ }^{13} \mathrm{C}-\mathrm{NMR}\right)$. Chemical shifts are given in ppm using the solvent itself as internal standard. The chemical shifts are expressed as $\square$ values and the coupling constants $(J)$ are given in Hertz. Mass spectra were recorded on a HP 5988A Quadrupol (EI ionisation, $70 \mathrm{eV}$ ) mass spectrometer. ESI and high-resolution mass spectra were recorded on a Bruker FTMS 4.7T BioAPEXII spectrometer. Absorption spectra were recorded with a Perkin-Elmer Lambda 40 
spectrometer. Photoirradiation was carrying out using a 50-500W Mercury arc lamp source from Thermo Oriel.

\section{Synthesis}

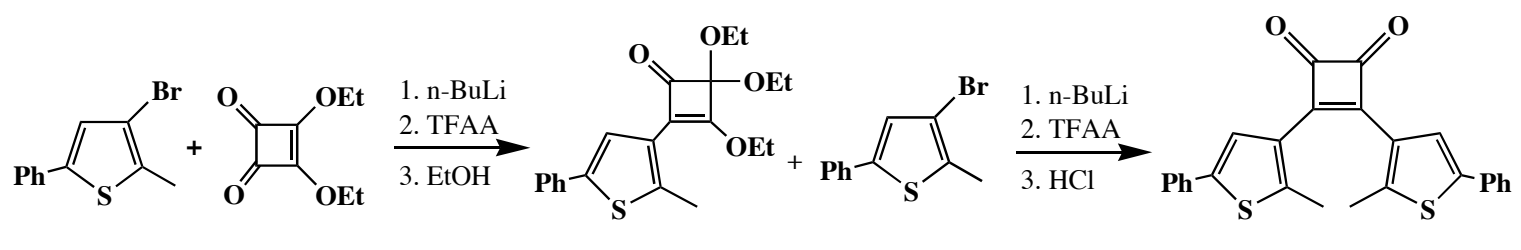

6

7

$1 \mathbf{a}$

\section{2,2-Diethoxy-4-(2-methyl-5-phenylthien-3-yl)-3-ethoxy cyclobuteneone (7)}

3-bromo-2-methyl-5-phenylthiophene ${ }^{\mathrm{S} 1} \mathbf{6}(1.31 \mathrm{~g}, 5.2 \mathrm{mmol})$ was dissolved in THF

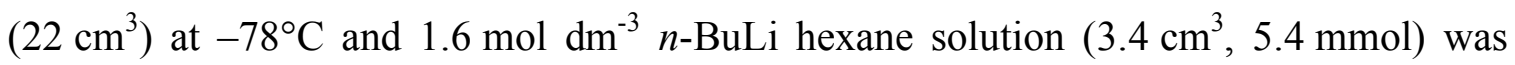
added slowly. The solution was stirred for $15 \mathrm{~min}$. and then added to a solution of diethylsquarate $(0.80 \mathrm{~g}, 4.7 \mathrm{mmol})$ in THF $\left(22 \mathrm{~cm}^{3}\right)$ via cannula. The solution was stirred for $25 \mathrm{~min}$ and then TFAA $\left(0.86 \mathrm{~cm}^{3}, 6.2 \mathrm{mmol}\right)$ was added. After stirring for $15 \mathrm{~min}$, EtOH $\left(1 \mathrm{~cm}^{3}, 17 \mathrm{mmol}\right)$ was added and the Dewar was removed. After warming to room temperature, the solvent was removed under vacuum and the residue was chromatographed on silica using hexane :Ethyl acetate $(4: 1)$ as eluent to give 7 (1.46 g, $83 \%) ; \square_{\mathrm{H}}\left(400 \mathrm{MHz}, \mathrm{CDCl}_{3}, \mathrm{CHCl}_{3}\right) 1.30(6 \mathrm{H}, \mathrm{t}, J 6.95), 1.51(3 \mathrm{H}, \mathrm{t}, J$ 7.07), $2.69(3 \mathrm{H}$, s), 3.77-3.85 (2 H, m), 3.86-3.95 (2 H, m), $4.62(2 \mathrm{H}, \mathrm{q}, J 7.07), 7.26(1 \mathrm{H}, \mathrm{tt}, J 7.42$ and 1.04), 7.36 (2 H, dd, $J 7.58$ and 7.58), $7.47(1 \mathrm{H}, \mathrm{s})$ and $7.56(2 \mathrm{H}, \mathrm{d}, J 7.83)$; $\left[\mathrm{l}\left(100 \mathrm{MHz}, \mathrm{CDCl}_{3}, \mathrm{CDCl}_{3}\right)\right.$ 15.1, 15.3, 15.4, 61.8, 69.6, 113.5, 123.2, 125.5, 126.0, 126.0, 127.3, 128.8, 134.0, 138.9, 140.9, 180.0 and $189.2 ; \mathrm{m} / z$ (EI) 372( $\left.\mathrm{M}^{+}, 13 \%\right)$, 343(53), 315(43), 241(95) and 213(100); $m / z(E S I): 395.1285\left(\mathrm{M}^{+}+\mathrm{Na} \mathrm{C}_{21} \mathrm{H}_{24} \mathrm{O}_{4} \mathrm{SNa}\right.$ requires 395.1288 ).

\section{3,4-Bis(2-methyl-5-phenylthien-3-yl)cyclobutenedione (1a)}

3-bromo-2-methyl-5-phenylthiophene ${ }^{\mathrm{S} 1}(1.01 \mathrm{~g}, 4.0 \mathrm{mmol})$ was dissolved in THF $\left(17 \mathrm{~cm}^{3}\right)$ at $-78^{\circ} \mathrm{C}$ and $1.6 \mathrm{~mol} \mathrm{dm} \mathrm{dm}^{-3} n$-BuLi hexane solution $\left(2.6 \mathrm{~cm}^{3}, 4.16 \mathrm{mmol}\right)$ was added slowly. The solution was stirred for $15 \mathrm{~min}$. and then added to a solution of 7 $(1.35 \mathrm{~g}, 3.49 \mathrm{mmol})$ in THF $\left(22 \mathrm{~cm}^{3}\right)$ via cannula. The solution was stirred for $25 \mathrm{~min}$ and then TFAA $\left(0.65 \mathrm{~cm}^{3}, 4.71 \mathrm{mmol}\right)$ was added. The solution was stirred for $20 \mathrm{~min}$ and then poured into a separatory funnel containing $10 \% \mathrm{HCl}\left(20 \mathrm{~cm}^{3}\right)$. The organic layer 
was separated and washed with $10 \% \mathrm{HCl}\left(2 \times 10 \mathrm{~cm}^{3}\right), \mathrm{H}_{2} \mathrm{O}\left(10 \mathrm{~cm}^{3}\right)$, brine $\left(20 \mathrm{~cm}^{3}\right)$ and then dried over $\mathrm{MgSO}_{4}$. The solvent was removed and the black solid was washed with ether to obtain 1a $(1.17 \mathrm{~g}, 79 \%)$; $\mathrm{Z}_{\mathrm{H}}\left(400 \mathrm{MHz}, \mathrm{CD}_{2} \mathrm{Cl}_{2}, \mathrm{CH}_{2} \mathrm{Cl}_{2}\right) 2.62(6 \mathrm{H}, \mathrm{s}), 7.31(2 \mathrm{H}$, tt, $J 7.3$ and 1.3), $7.37(2 \mathrm{H}, \mathrm{dd}, J 7.6$ and 7.1$), 7.46(2 \mathrm{H}, \mathrm{s})$ and $7.55(2 \mathrm{H}, \mathrm{d}, J 7.1$ and $1.3)$; $\square\left(\mathrm{l}\left(100 \mathrm{MHz}, \mathrm{CD}_{2} \mathrm{Cl}_{2}, \mathrm{CH}_{2} \mathrm{Cl}_{2}\right)\right.$ 16.2, 122.6, 126.0, 128.2, 128.5, 129.4, 133.5, 143.1, 147.1, 183.8 and 195.9; $\mathrm{m} / z$ (EI) 426(M $\left.\mathrm{M}^{+}, 31 \%\right), 370(100), 185(8) ; \mathrm{m} / z$ (ESI) : $427.0823\left(\mathrm{M}^{+}+\mathrm{H} . \mathrm{C}_{26} \mathrm{H}_{19} \mathrm{O}_{2} \mathrm{~S}_{2}\right.$ requires 427.0821).

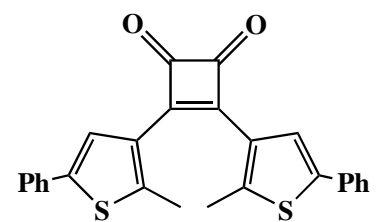

$1 \mathbf{a}$

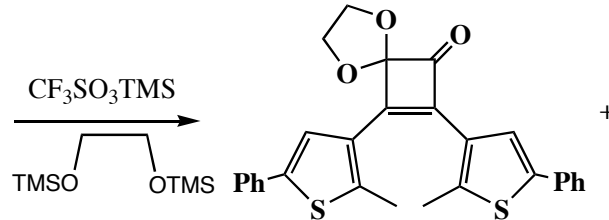

$2 \mathbf{a}$

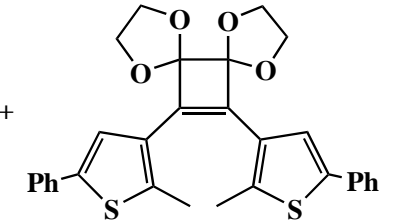

$3 \mathbf{a}$

3,4-Bis(2-methyl-5-phenylthien-3-yl)cyclobutenedione Mono(ethylene ketal) (2a) and 3,4-Bis(2-methyl-5-phenylthien-3-yl)cyclobutenedione Bis(ethylene ketal) (3a)

To 3,4-Bis(2-methyl-5-phenylthien-3-yl)cyclobutenedione $1 \mathbf{a}(100 \mathrm{mg}, 0.24 \mathrm{mmol})$ and 1,2-bis[(trimethylsilyl)oxy]ethane $\quad\left(2 \mathrm{~cm}^{3}, \quad 13.6 \mathrm{mmol}\right), \quad$ trimethylsilyl trifluoromethanesulfonate $\left(0.23 \mathrm{~cm}^{3}, 1.25 \mathrm{mmol}\right)$ was added under argon. The solution was heated at $80^{\circ} \mathrm{C}$ for $1 \mathrm{~h}$. After cooling, ether was added. The solution was filtered and the precipitate washed with ether and the solid was chromatographed on basic aluminum oxide using dichloromethane: hexane $(1: 1)$ as eluent to give $\mathbf{2 a}(19 \mathrm{mg}, 17 \%)$ $\square_{\mathrm{H}}\left(400 \mathrm{MHz}, \mathrm{CDCl}_{3}, \mathrm{CHCl}_{3}\right) 2.37(3 \mathrm{H}, \mathrm{s}) 2.44(3 \mathrm{H}, \mathrm{s}) 4.26(2 \mathrm{H}, \mathrm{m}) 4.33(2 \mathrm{H}, \mathrm{m}) 7.29$ $(3 \mathrm{H}, \mathrm{m}) 7.37(5 \mathrm{H}, \mathrm{m})$ and $7.53(4 \mathrm{H}, \mathrm{m}$,$) ; \mathrm{L}\left(100 \mathrm{MHz}, \mathrm{CDCl}_{3}, \mathrm{CDCl}_{3}\right)$ 15.2, 15.6, 66.3, 120.0, 122.7, 123.1, 125.6, 125.7, 127.6, 127.7, 127.9, 128.9, 129.0, 129.3, 133.4, 133.7, $141.3,141.5,142.0,144.1,149.0,165.3$ and $194.0 ; \mathrm{m} / \mathrm{z}$ (EI): surprisingly immeasurable and 3a $(80 \mathrm{mg}, 65 \%)$. $\mathrm{Z}_{\mathrm{H}}\left(400 \mathrm{MHz}, \mathrm{CD}_{2} \mathrm{Cl}_{2}, \mathrm{CH}_{2} \mathrm{Cl}_{2}\right) 2.21(6 \mathrm{H}, \mathrm{s}), 3.95-4.04(4 \mathrm{H}, \mathrm{m})$, 4.08-4.18 (4 H, m), $7.26(2 \mathrm{H}, \mathrm{tt}, J 7.3$ and 1.3), $7.30(2 \mathrm{H}, \mathrm{s}), 7.35(4 \mathrm{H}, \mathrm{dd}, J 8.3$ and 7.3) and 7.54 (4 H, dd, $J 8.4$ and 1.3); $Z_{e}\left(100 \mathrm{MHz}, \mathrm{CD}_{2} \mathrm{Cl}_{2}, \mathrm{CH}_{2} \mathrm{Cl}_{2}\right)$ 14.9, 65.8, 114.7, $123.5,125.8,127.8,129.2,130.8,134.4,140.1,140.3$ and $141.2 ; m / z(E I): 514\left(\mathrm{M}^{+}\right.$, $30 \%), 426(38)$ and $370(100) ; m / z(E S I): 537.1163\left(\mathrm{M}^{+}+\mathrm{Na} . \mathrm{C}_{30} \mathrm{H}_{26} \mathrm{O}_{4} \mathrm{~S}_{2} \mathrm{Na}\right.$ requires 537.1164). 


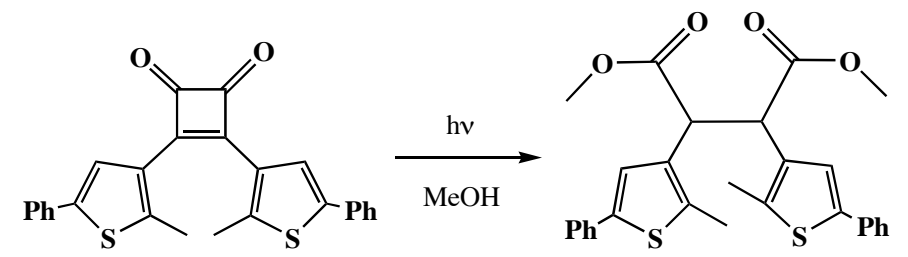

\section{Dimethyl 2,3-(2-methyl-5-phenylthien-3-yl)-1,4-butanedioate (5)}

1a $(10 \mathrm{mg}, 23 \square \mathrm{mol})$ was dissolved in quartz reactor in dichloromethane: methanol $\left(2 \mathrm{~cm}^{3}, 1: 1\right)$ as solvent. Oxygen was removed by bubbling argon and then the solution was irradiated at $365 \mathrm{~nm}$ until the yellow color was completely disappeared. Solvents were removed and the residue was suspended in Hexane/Ethyl acetate $\left(3 \mathrm{~cm}^{3}, 5: 1\right)$. The precipitate was filtered to get $5(7 \mathrm{mg}, 61 \%)$. $\mathrm{ZH}_{\mathrm{H}}\left(400 \mathrm{MHz}, \mathrm{CDCl}_{3}, \mathrm{CHCl}_{3}\right) 2.56(6 \mathrm{H}, \mathrm{s})$, $3.51(6 \mathrm{H}, \mathrm{s}), 4.47(2 \mathrm{H}, \mathrm{s}), 7.26(2 \mathrm{H}, \mathrm{t}, J 7.33), 7.37(6 \mathrm{H}, \mathrm{m})$ and $7.58(4 \mathrm{H}, \mathrm{d}, J 7.33)$; ${ }_{\mathrm{C}}\left(100 \mathrm{MHz}, \mathrm{CDCl}_{3}, \mathrm{CHCl}_{3}\right)$ 13.2, 47.8, 52.1, 122.3, 125.5, 127.2, 128.8, 132.5, 134.3, 136.6, 140.4 and 171.6; $\mathrm{m} / \mathrm{z}(\mathrm{EI}) 491\left(\mathrm{M}^{+}, 10 \%\right), 245(100)$ and $213(100) ; \mathrm{m} / \mathrm{z}(\mathrm{ESI})$ : $513.1159\left(\mathrm{M}^{+}+\mathrm{Na} . \mathrm{C}_{28} \mathrm{H}_{26} \mathrm{O}_{4} \mathrm{~S}_{2} \mathrm{Na}\right.$ requires 513.1164).

\section{Thermal stability}

Open-ring reaction of $\mathbf{1 b}$ occurs spontaneously at room temperature. The intensity of the characteristic peak at $800 \mathrm{~nm}$ decreases and this intensity was measured at different time.

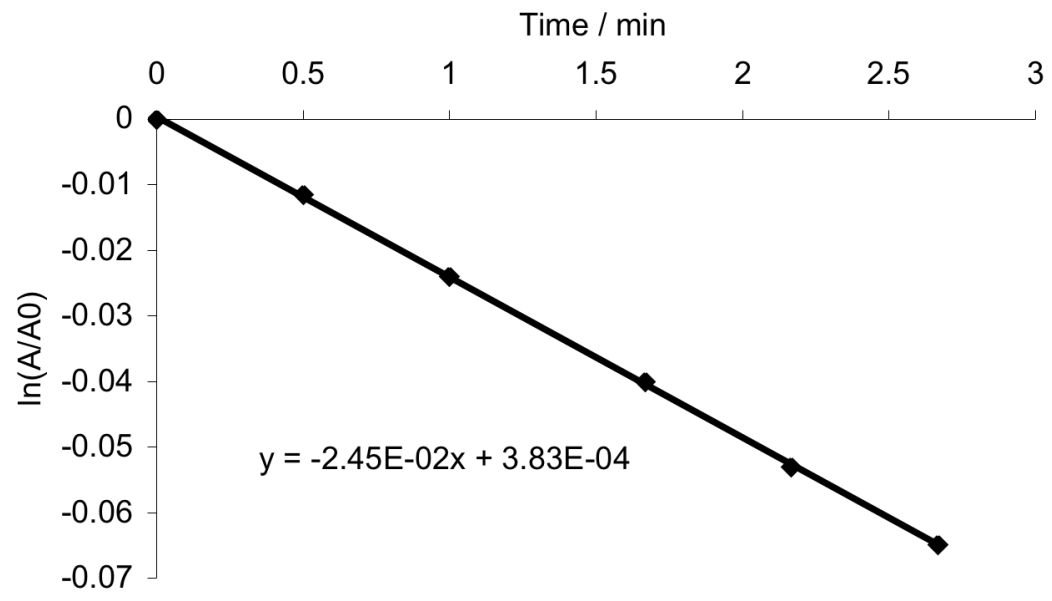

Fig. S1 : Open-ring reaction of $\mathbf{1 b}$ modeling by a first order reaction 
The slope in the figure S1 allows calculating the rate constant and the half-lifetime $t_{1 / 2}$.

$$
t_{1 / 2}=\frac{\ln (2)}{k}=\frac{\ln (2)}{0.0245}=28 h^{\square 1}
$$

\section{Fatigue resistance}

Fatigue resistance was measured as following: In deaerated acetonitrile solution, the dithienylethene derivative was irradiated till $90 \%$ of the photostationary state was reached and the colored closed form is completely bleached. The sequence was repeated several times. After each cycle, absorbance of the colored compound is measured.

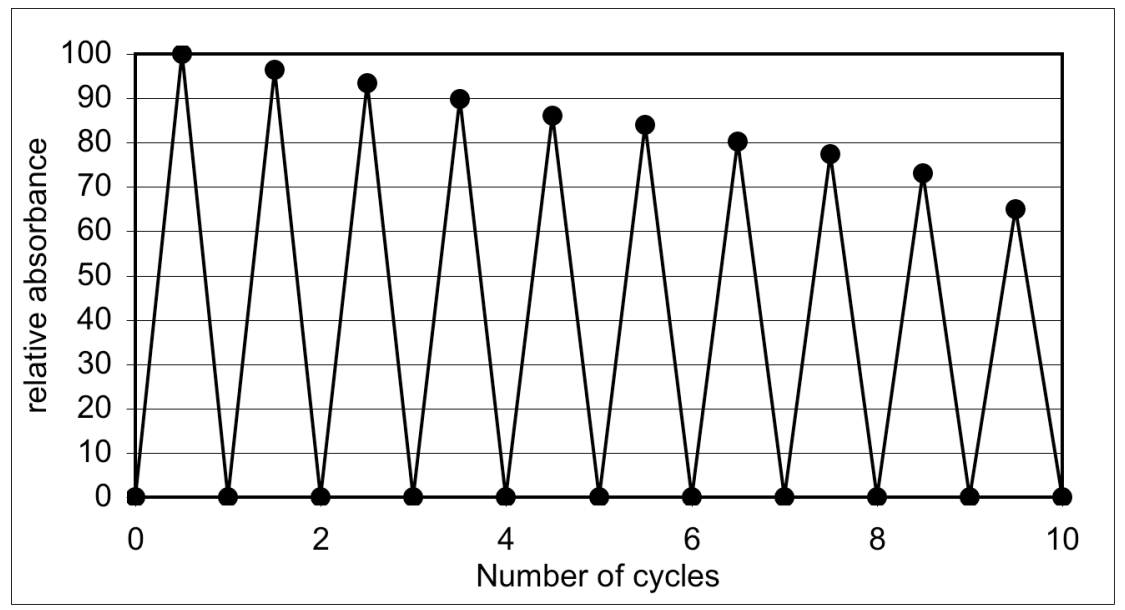

Fig. S2 : Photofatigue resistance of the photochromic system 3

\section{Crystal structure}

A yellow crystal of compound 1a was mounted on a Stoe Imaging Plate Diffractometer System (Stoe \& Cie, 1995) equipped with a one-circle $\varphi$ goniometer and a graphitemonochromator. Data collection was performed at $-100^{\circ} \mathrm{C}$ using $\mathrm{Mo}-\mathrm{K} \alpha$ radiation $(\lambda=$ $0.71073 \AA$ ). 133 exposures (10 min per exposure) were obtained at an image plate distance of $70 \mathrm{~mm}$ with $0<\varphi<199.5^{\circ}$ and with the crystal oscillating through $1.5^{\circ}$ in $\varphi$. The resolution was $\mathrm{D}_{\min }-\mathrm{D}_{\max } 12.45-0.81 \AA$. 
This compound crystallized in a monoclinic cell (P 2 $/ \mathrm{n}$ ). The molecular formula of this compound is $\left(\mathrm{C}_{26} \mathrm{H}_{18} \mathrm{O}_{2} \mathrm{~S}_{2}\right)$.
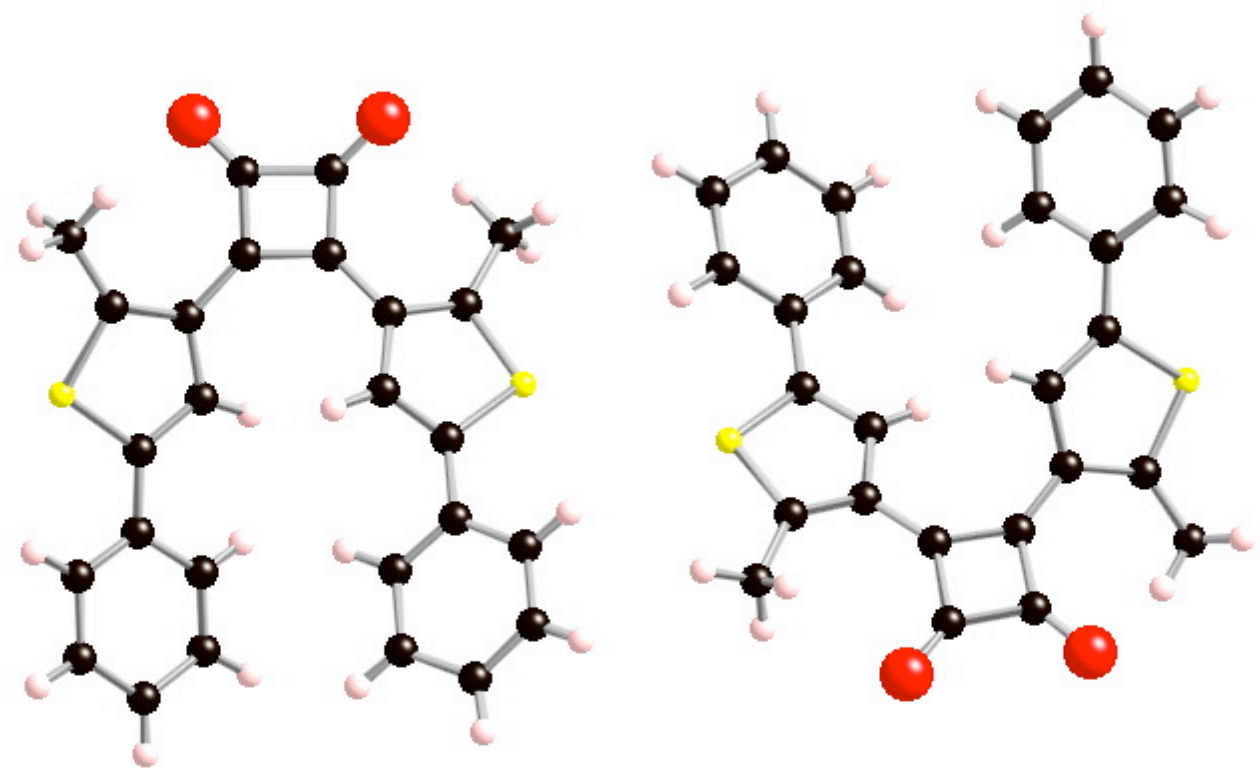

Fig. S3 : Crystal structure of 1a 
6. ${ }^{1} \mathrm{H}-\mathrm{NMR}$ and ${ }^{13} \mathrm{C}-\mathrm{NMR}$

2,2-Diethoxy-4-(2-methyl-5-phenylthien-3-yl)-3-ethoxy cyclobuteneone (7)

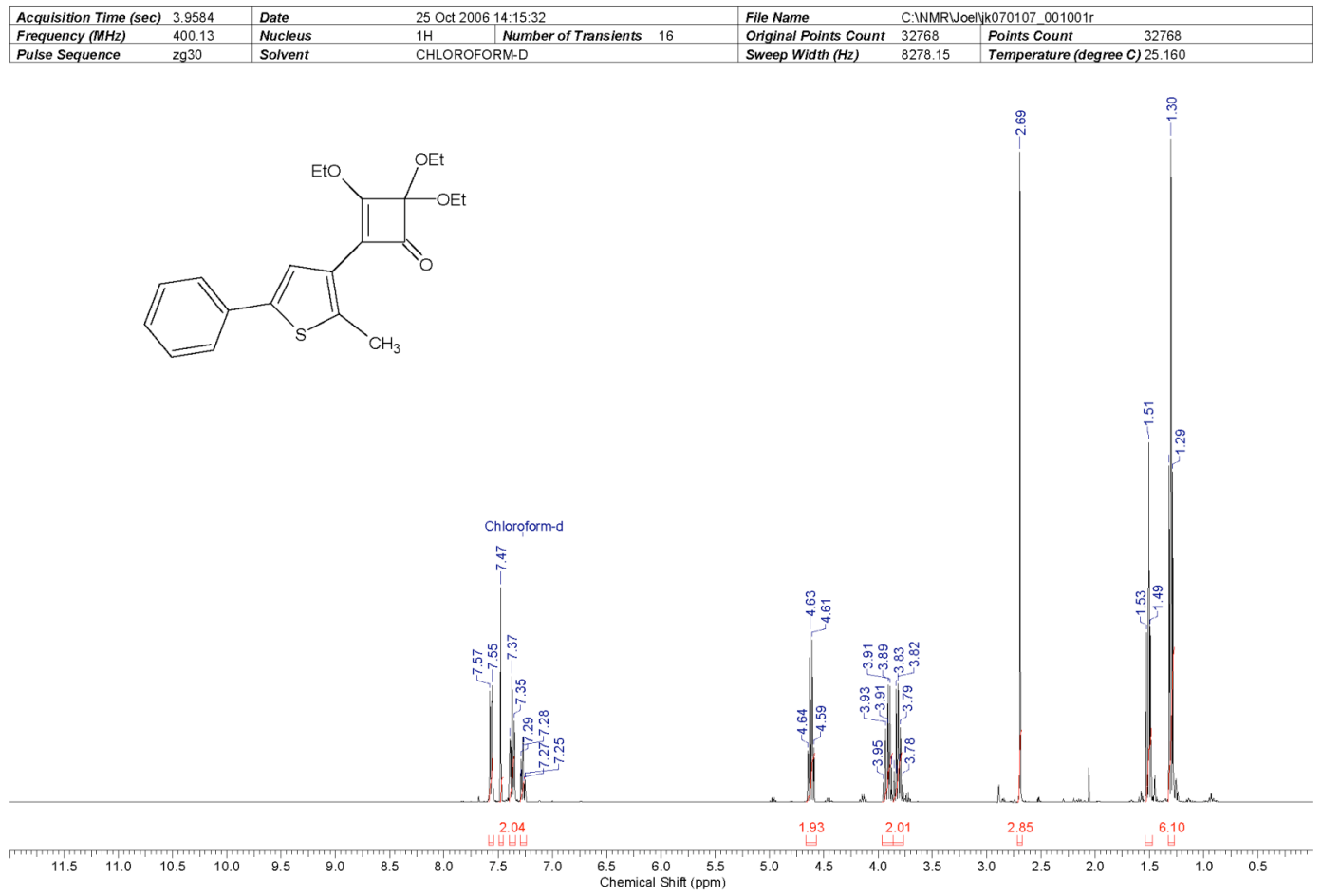



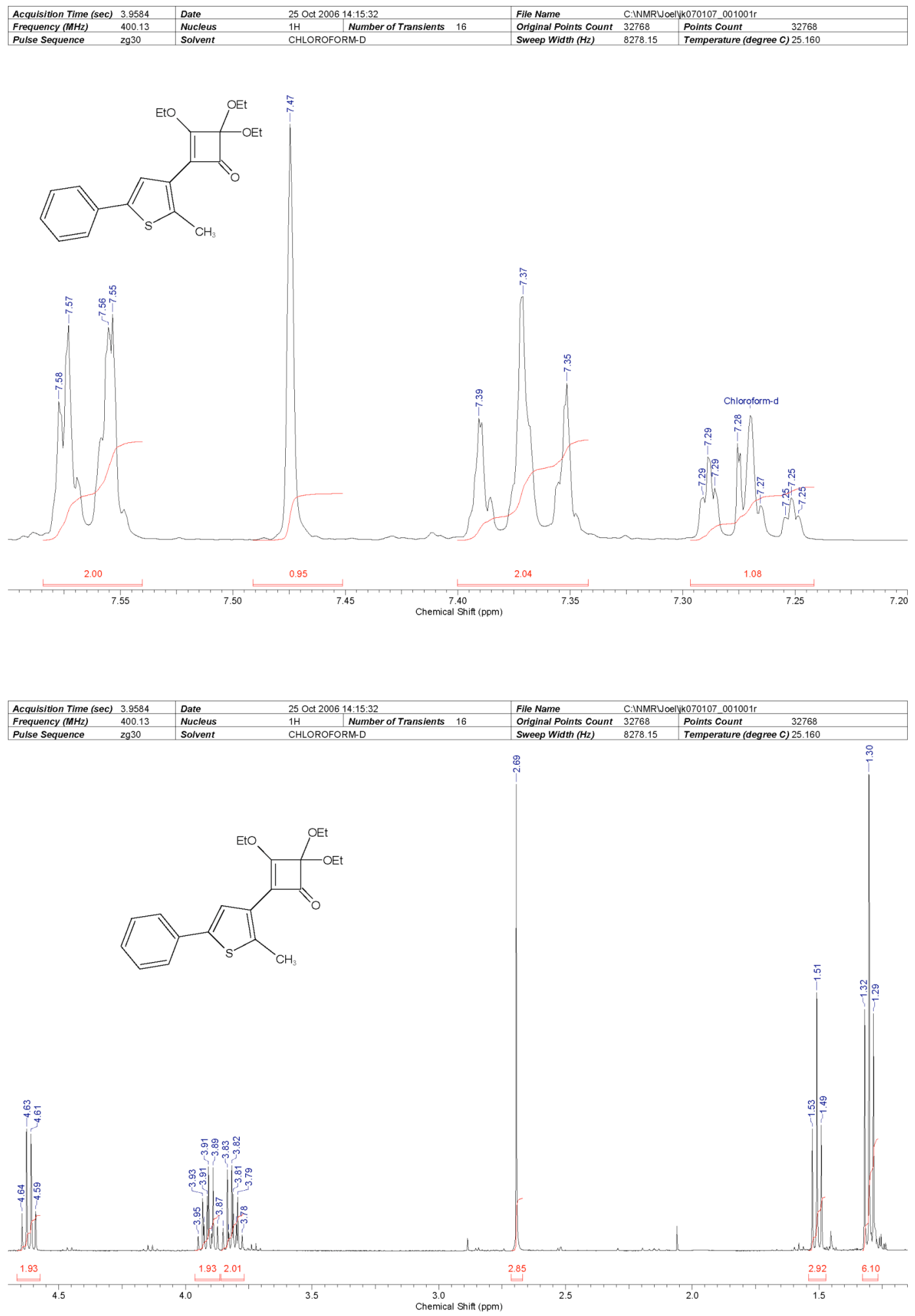

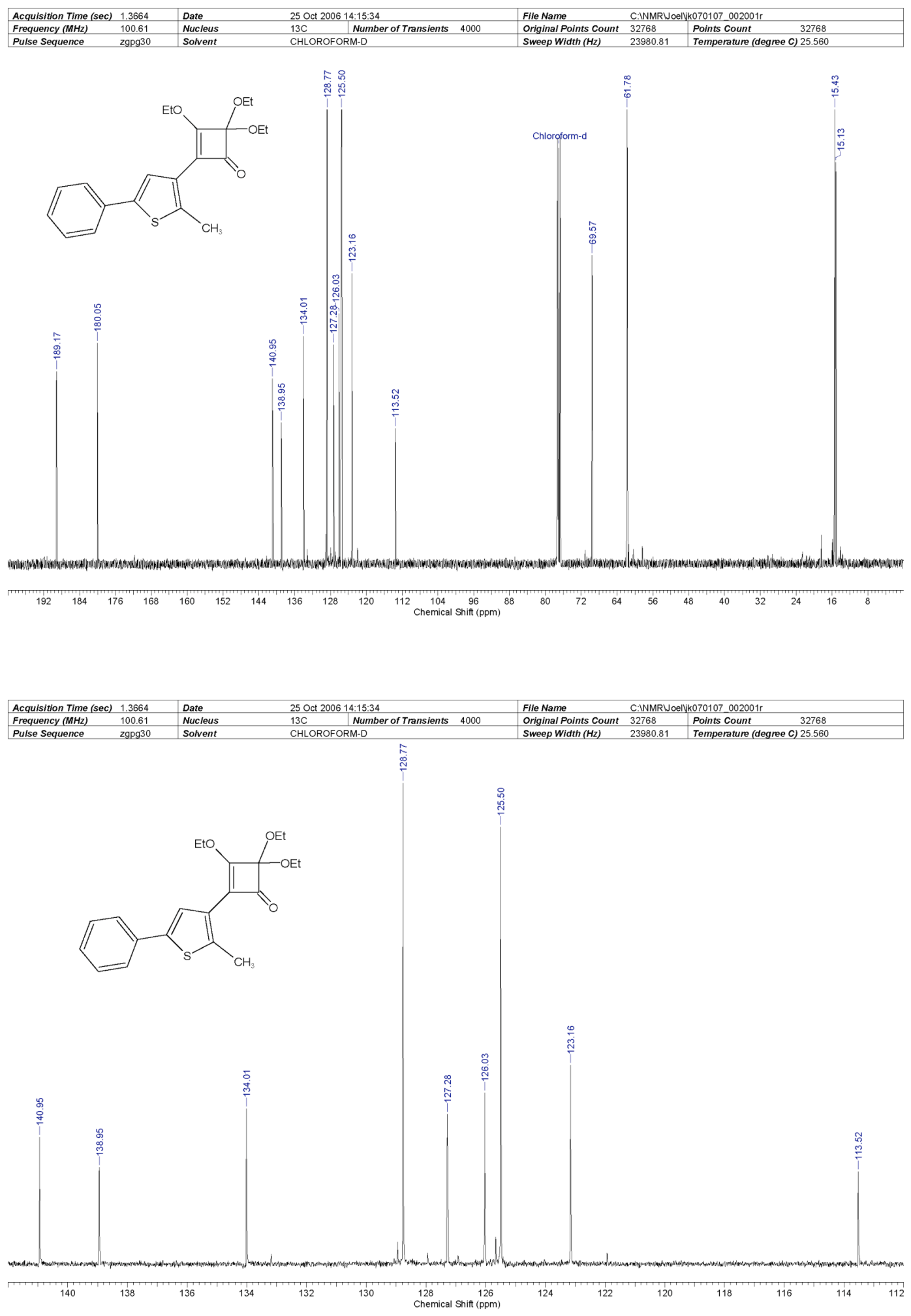


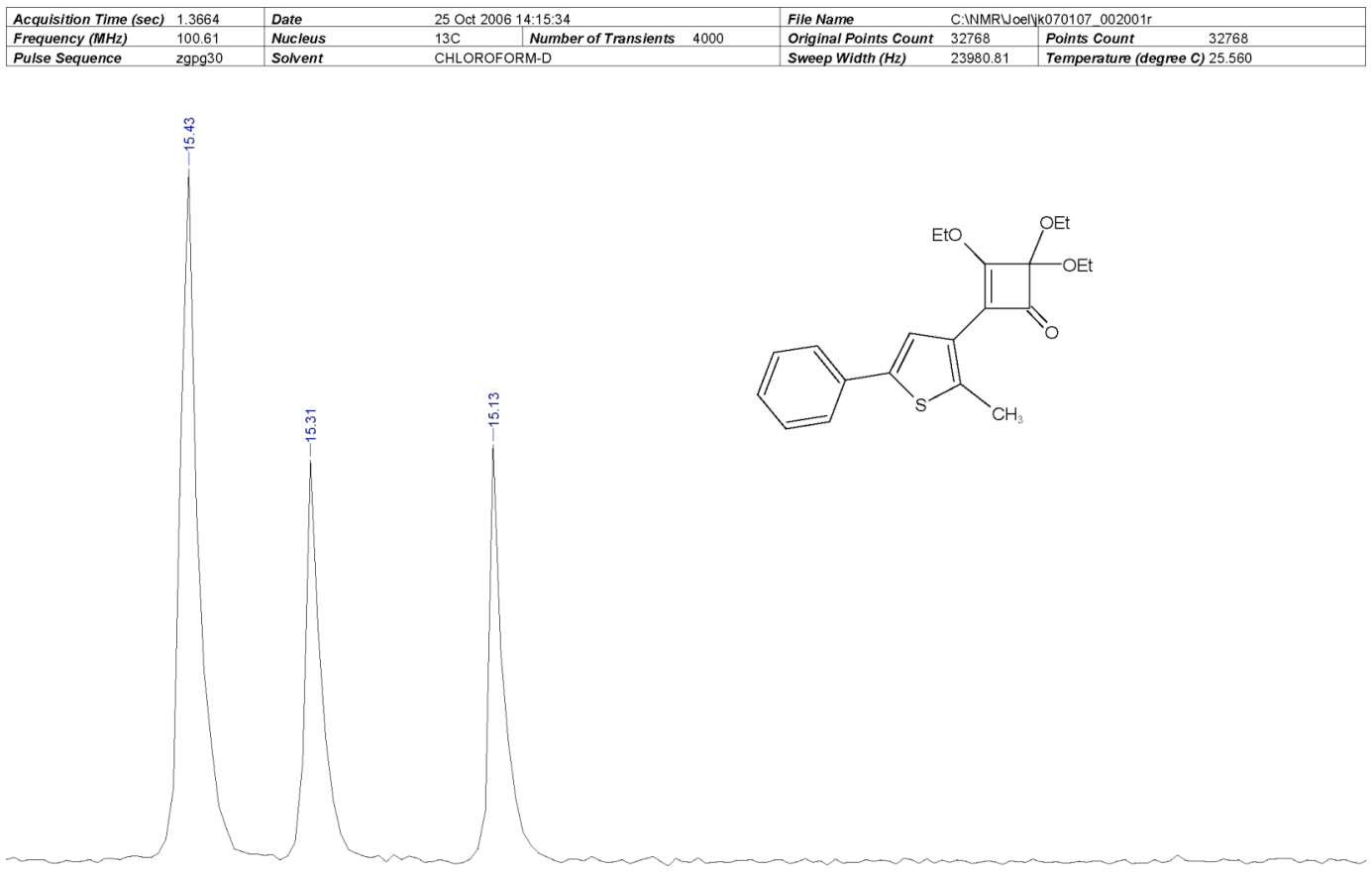

$\begin{array}{lllllllllllllllll}15.55 & 15.50 & 15.45 & 15.40 & 15.35 & 15.30 & 15.25 & 15.20 & 15.15 & 15.10 & 15.05 & \begin{array}{l}15.50 \\ \text { Chemical shit (ppm) }\end{array}\end{array}$ 


\section{3,4-Bis(2-methyl-5-phenylthien-3-yl)cyclobutenedione (1a)}

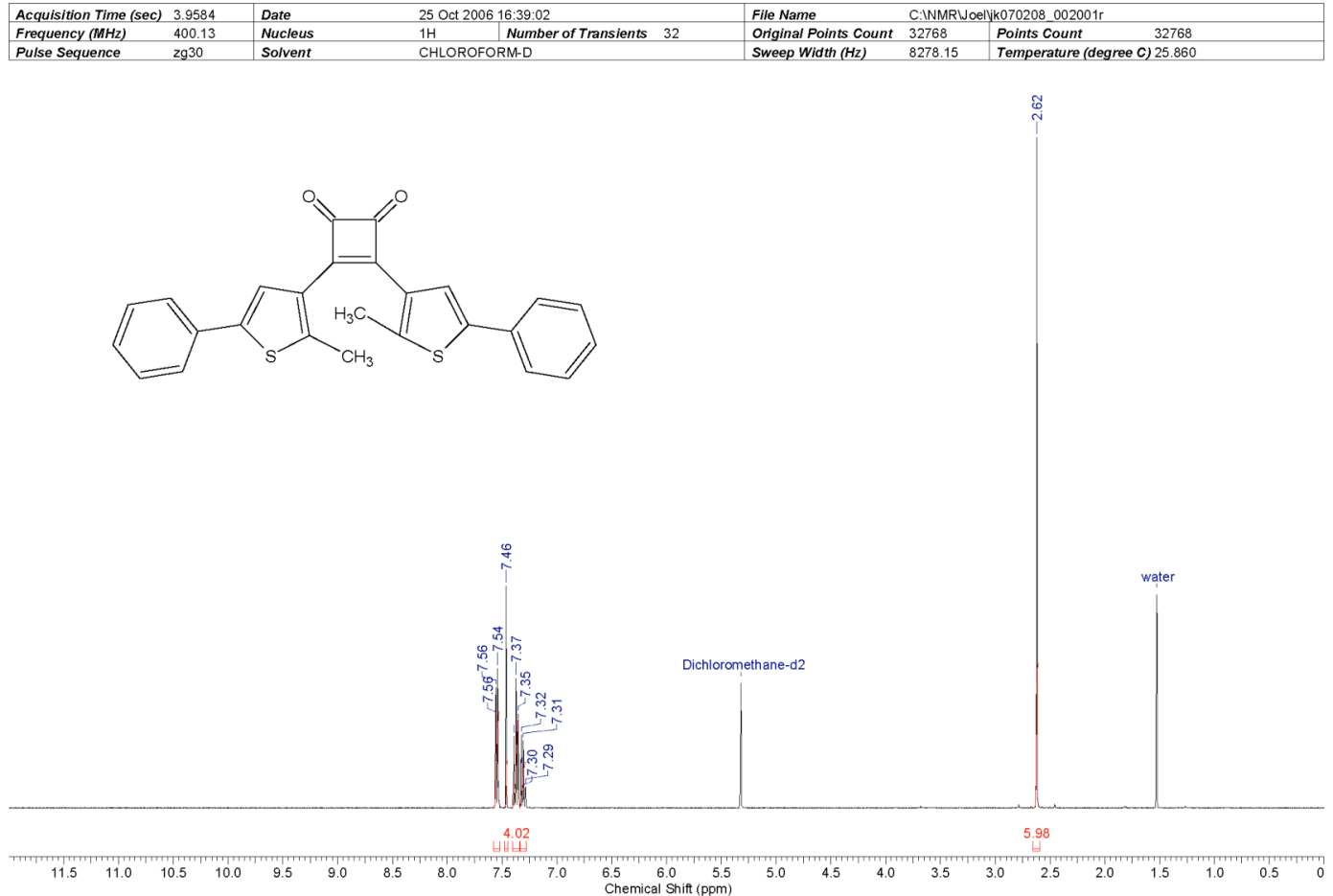

\begin{tabular}{|c|c|c|c|c|c|c|}
\hline \multirow{2}{*}{\multicolumn{2}{|c|}{$\begin{array}{ll}\text { Acquisition Time (sec) } & 3.9584 \\
\text { Frequency (MHz) } & 400.13\end{array}$}} & \multirow{2}{*}{$\begin{array}{l}\text { Date } \\
\text { Nucleus }\end{array}$} & 25 Oct $200616: 39: 02$ & \multicolumn{3}{|c|}{ C:INMRWoellik070208 002001r } \\
\hline & & & Number of Transients 32 & Original Points Count & 32768 & Points Count \\
\hline Pulse Sequence & $\mathrm{zg} 30$ & Solvent & CHLOROFORM-D & Sweep Width (Hz) & 8278.15 & Temperature (degree $C$ ) 25.860 \\
\hline
\end{tabular}

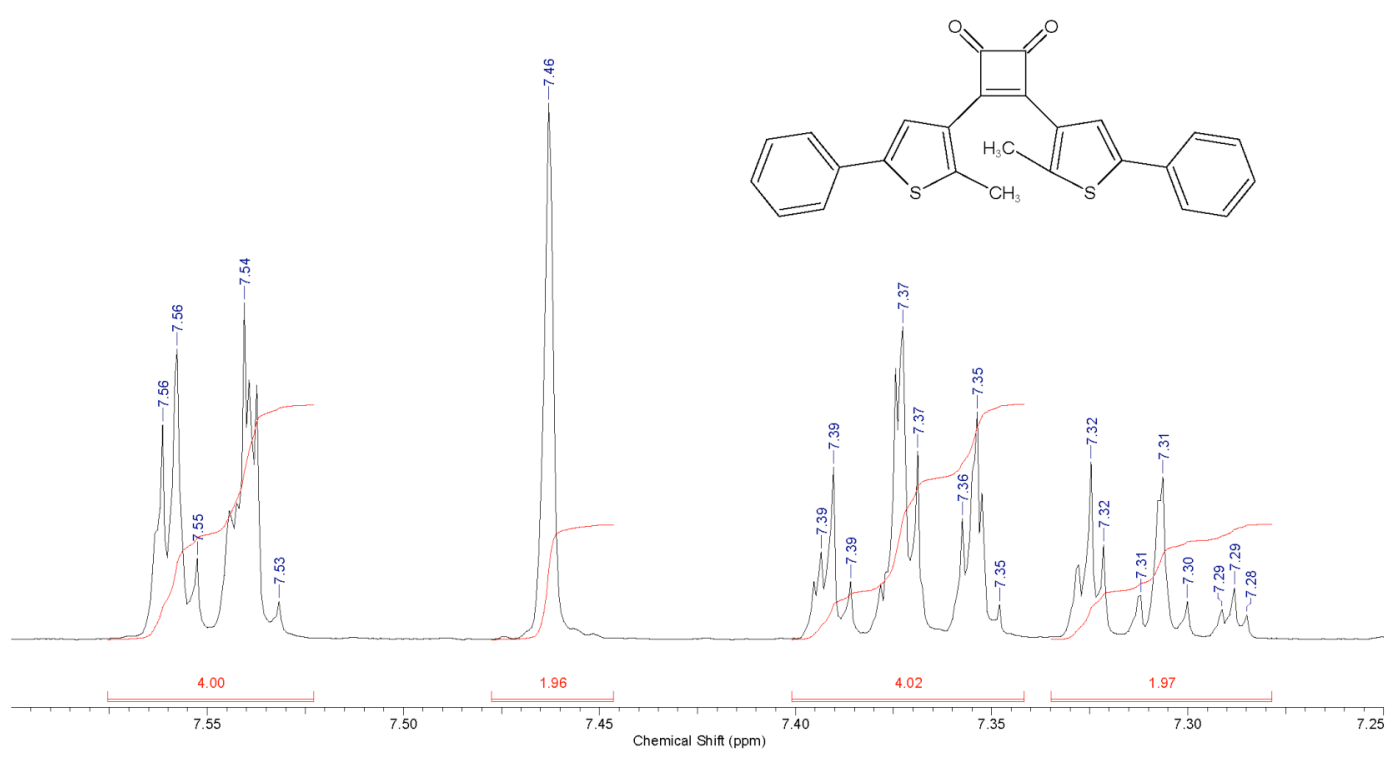



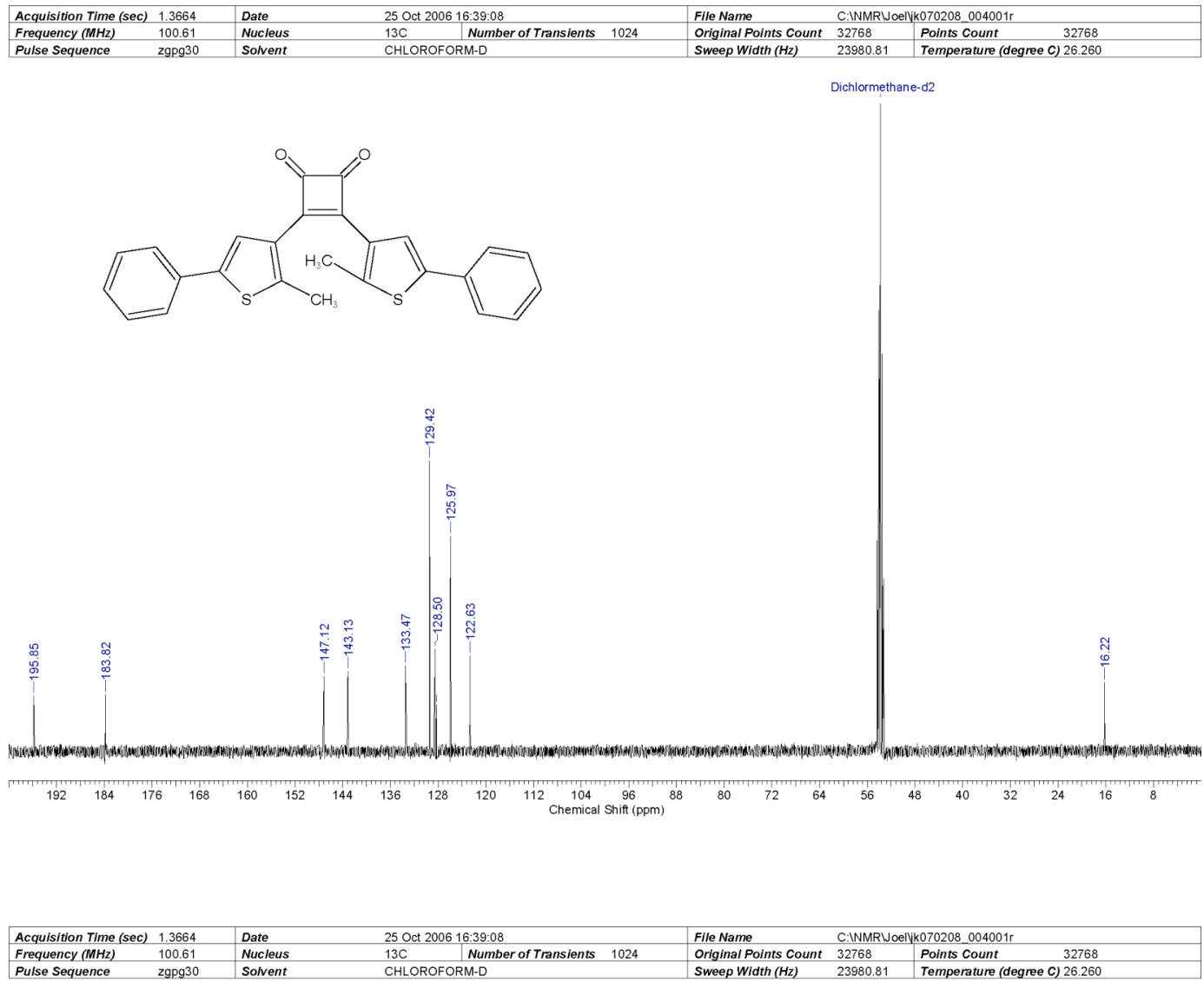

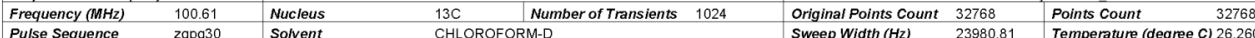
Puse
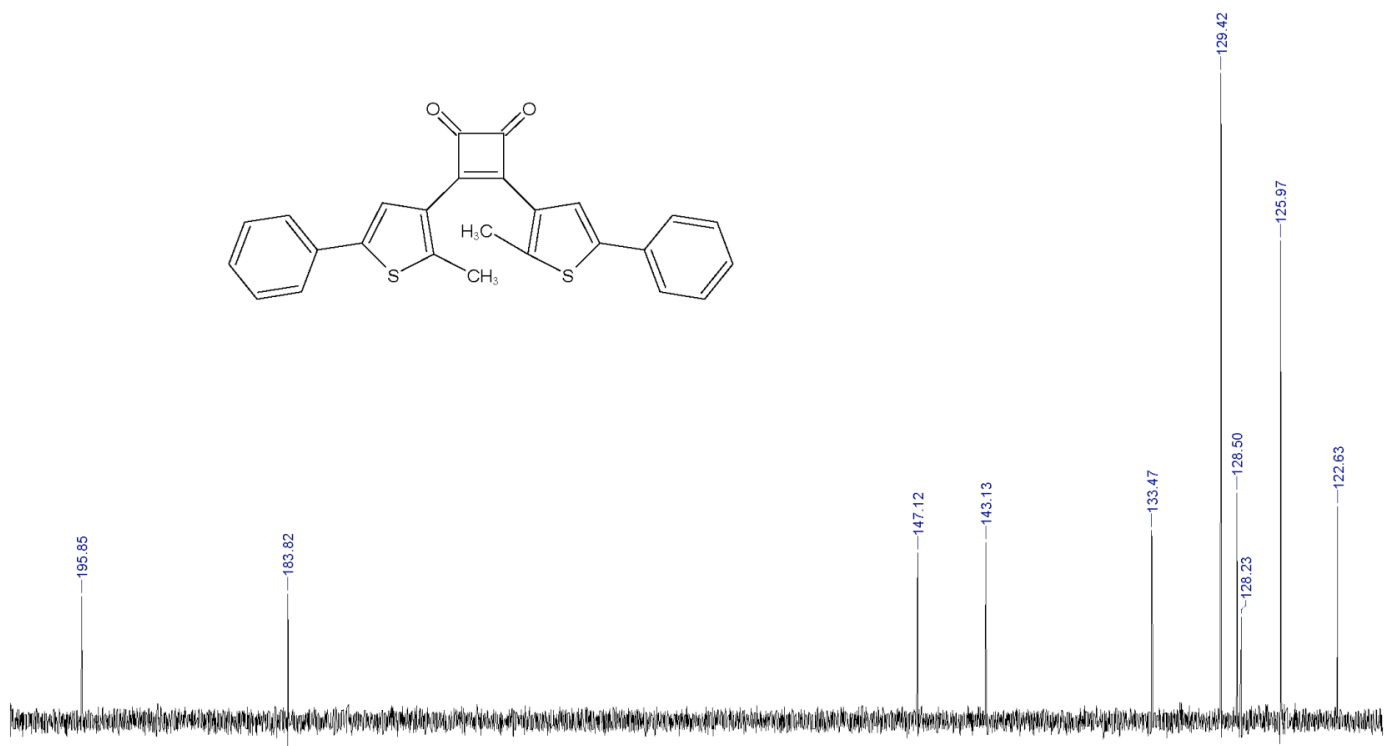
185
$180+175$
170
$\begin{array}{lll}165 & 160 \\ \text { Chemical Shitt (ppm) } & 155\end{array}$
$150 \quad 14$
$140 \quad 135130$
$125 \quad 120$ 
3,4-Bis(2-methyl-5-phenylthien-3-yl)cyclobutenedione Mono(ethylene ketal) (2a)
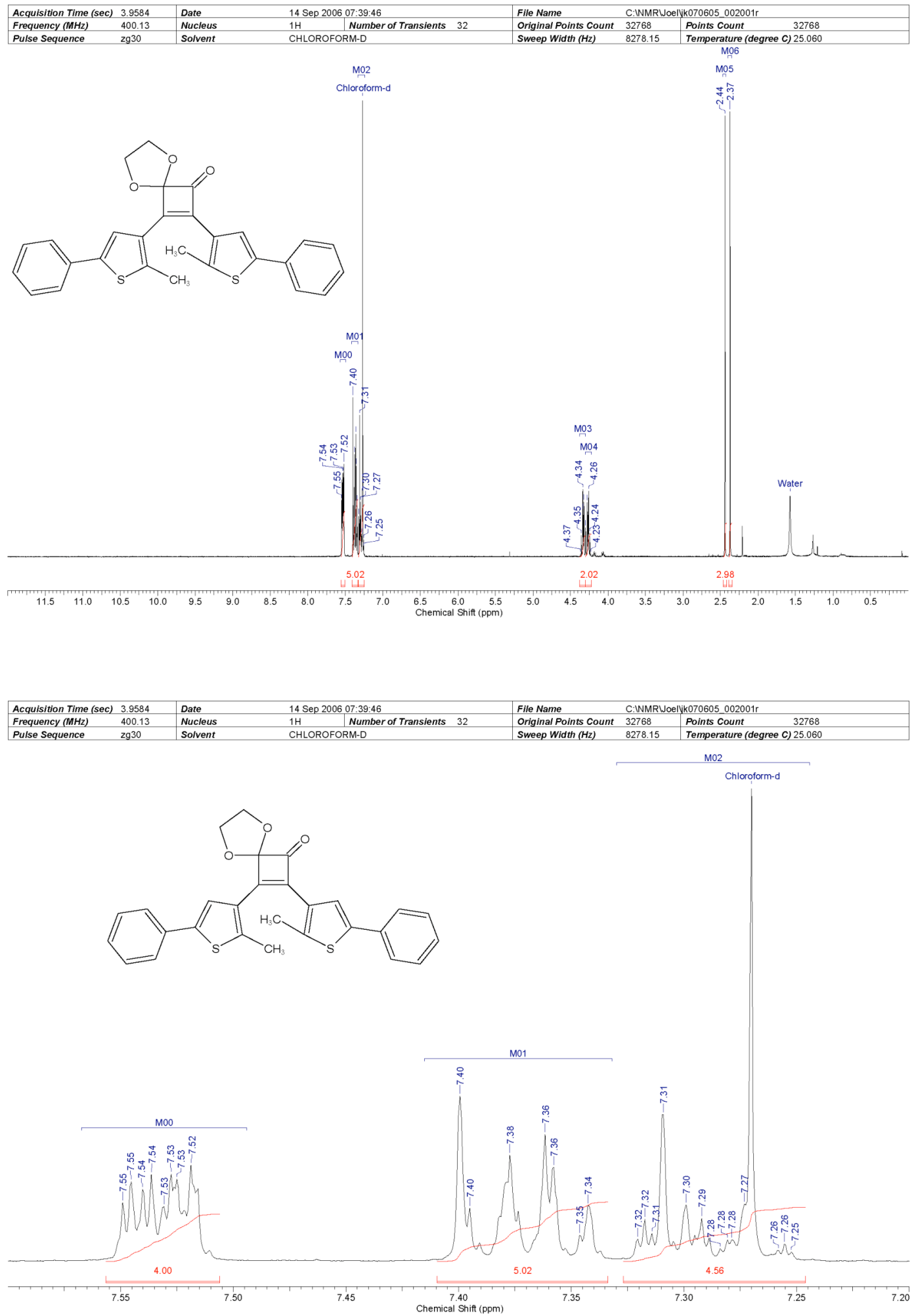

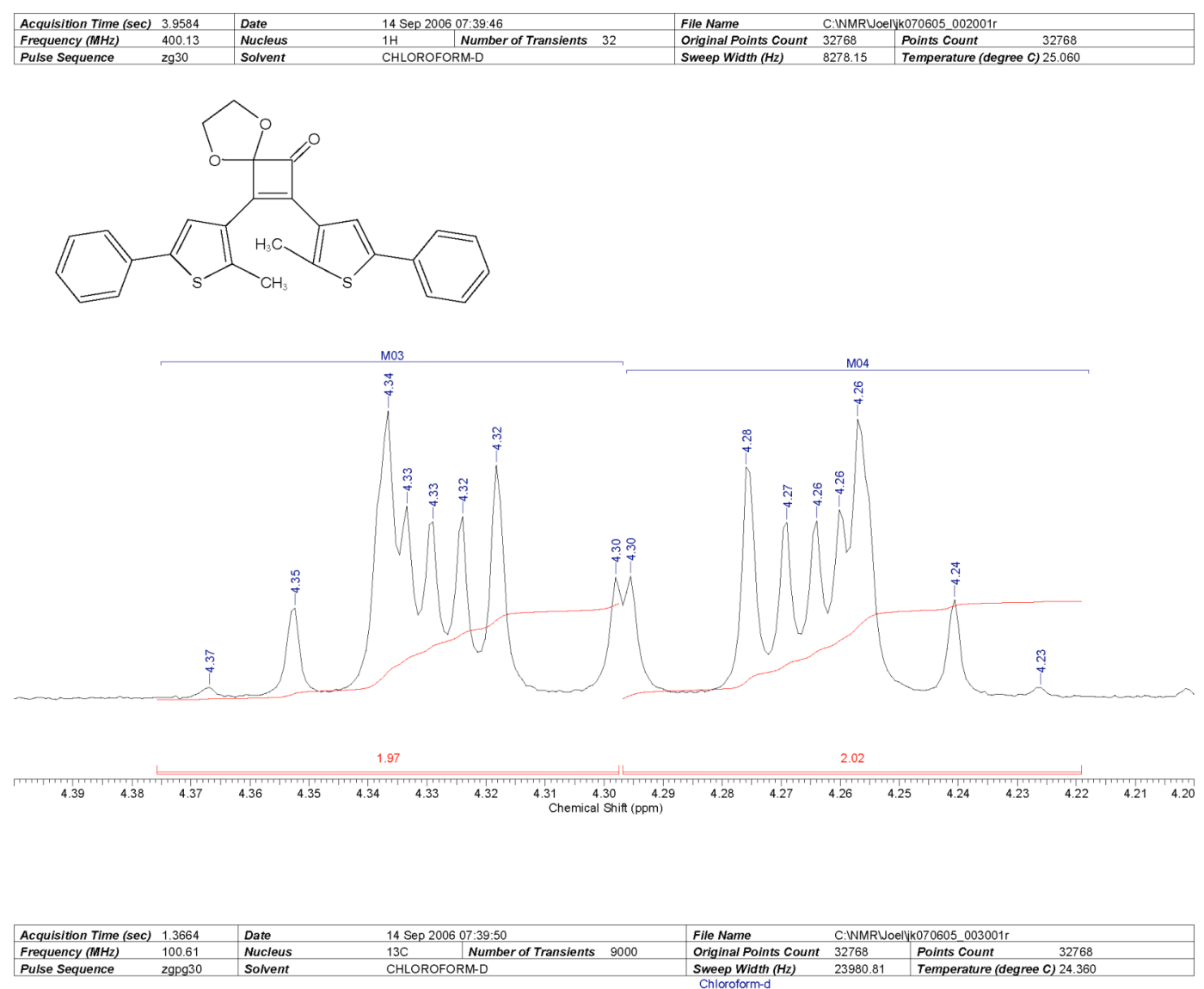

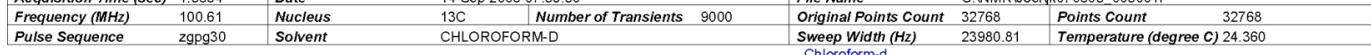
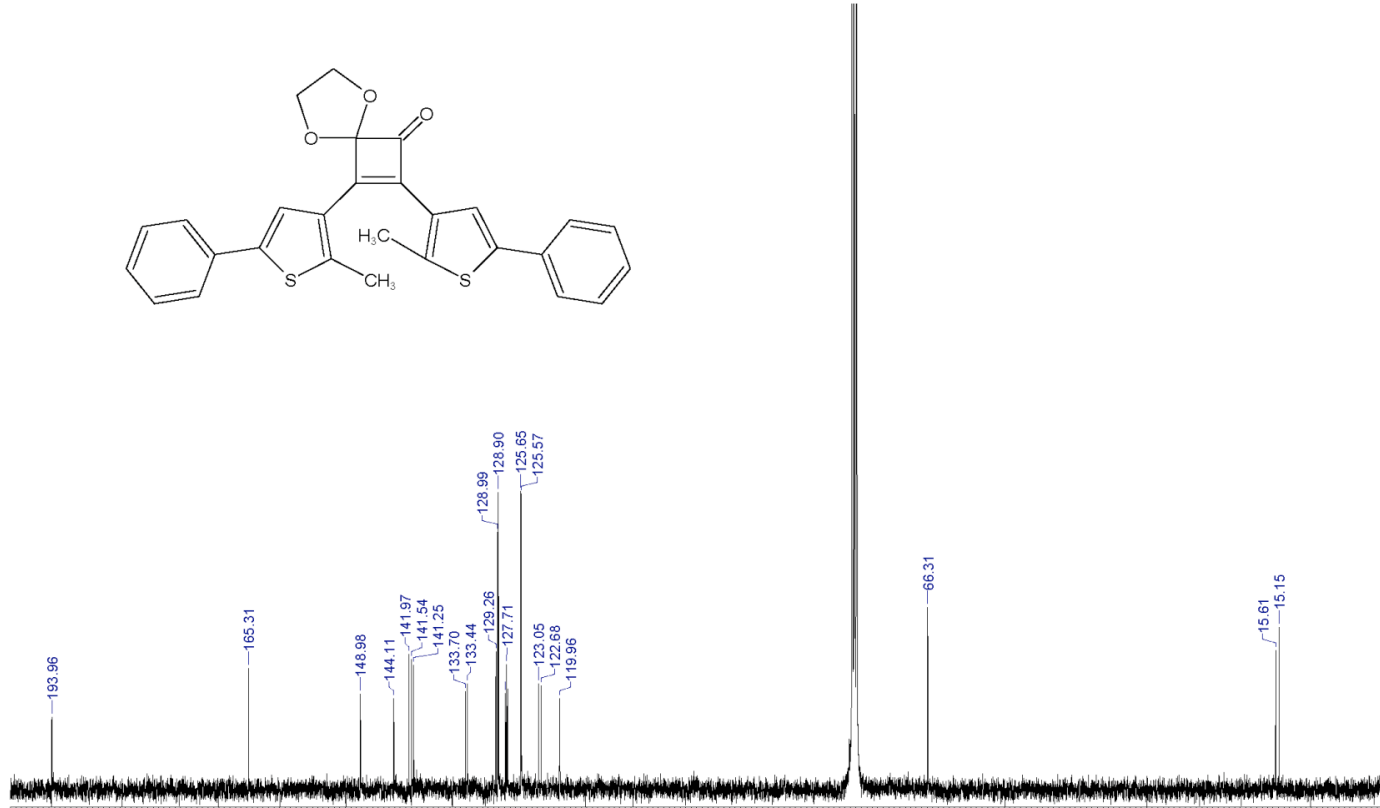

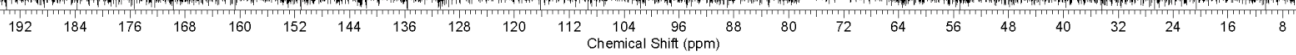




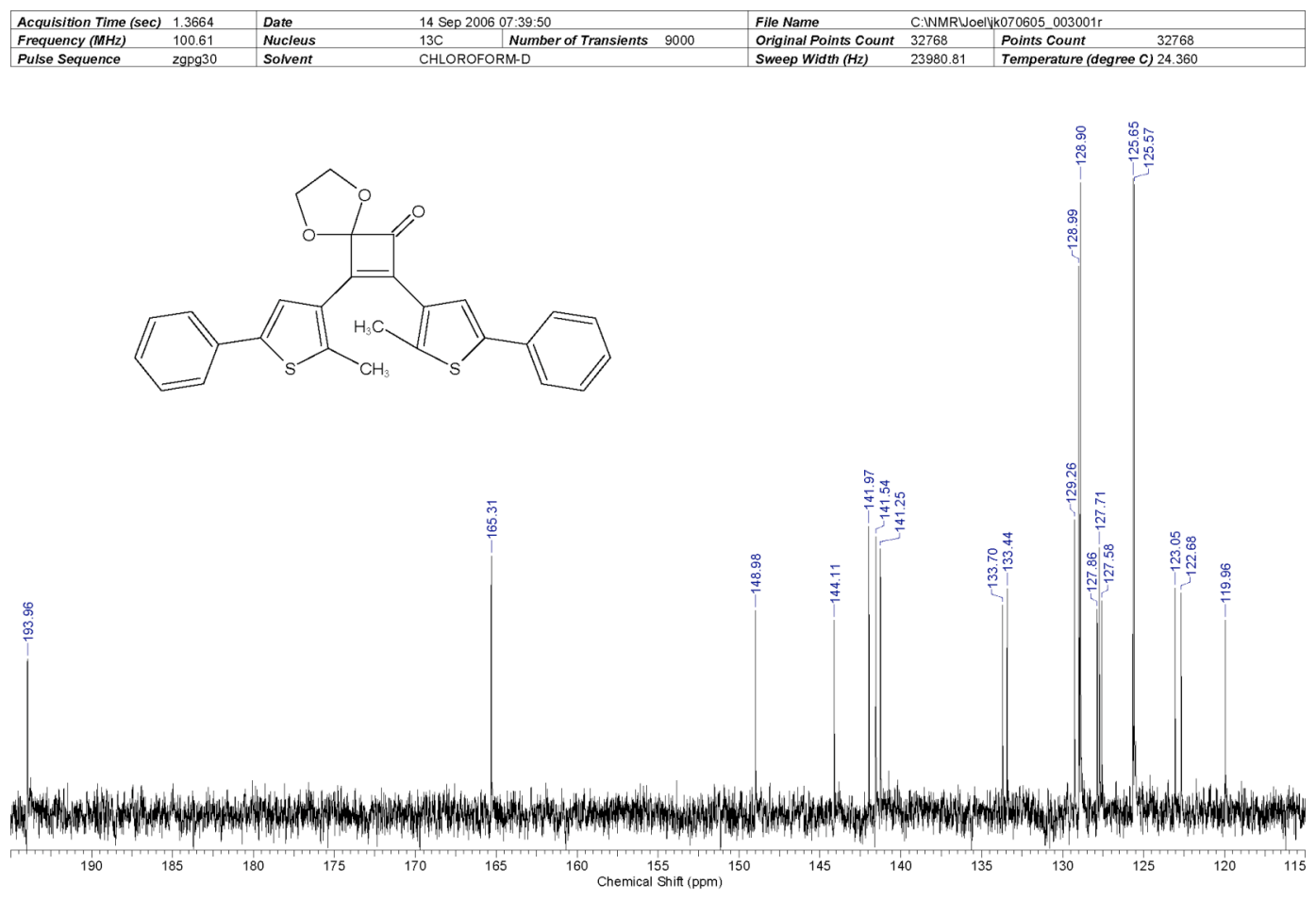




\section{3,4-Bis(2-methyl-5-phenylthien-3-yl)cyclobutenedione Bis(ethylene ketal) (3a)}

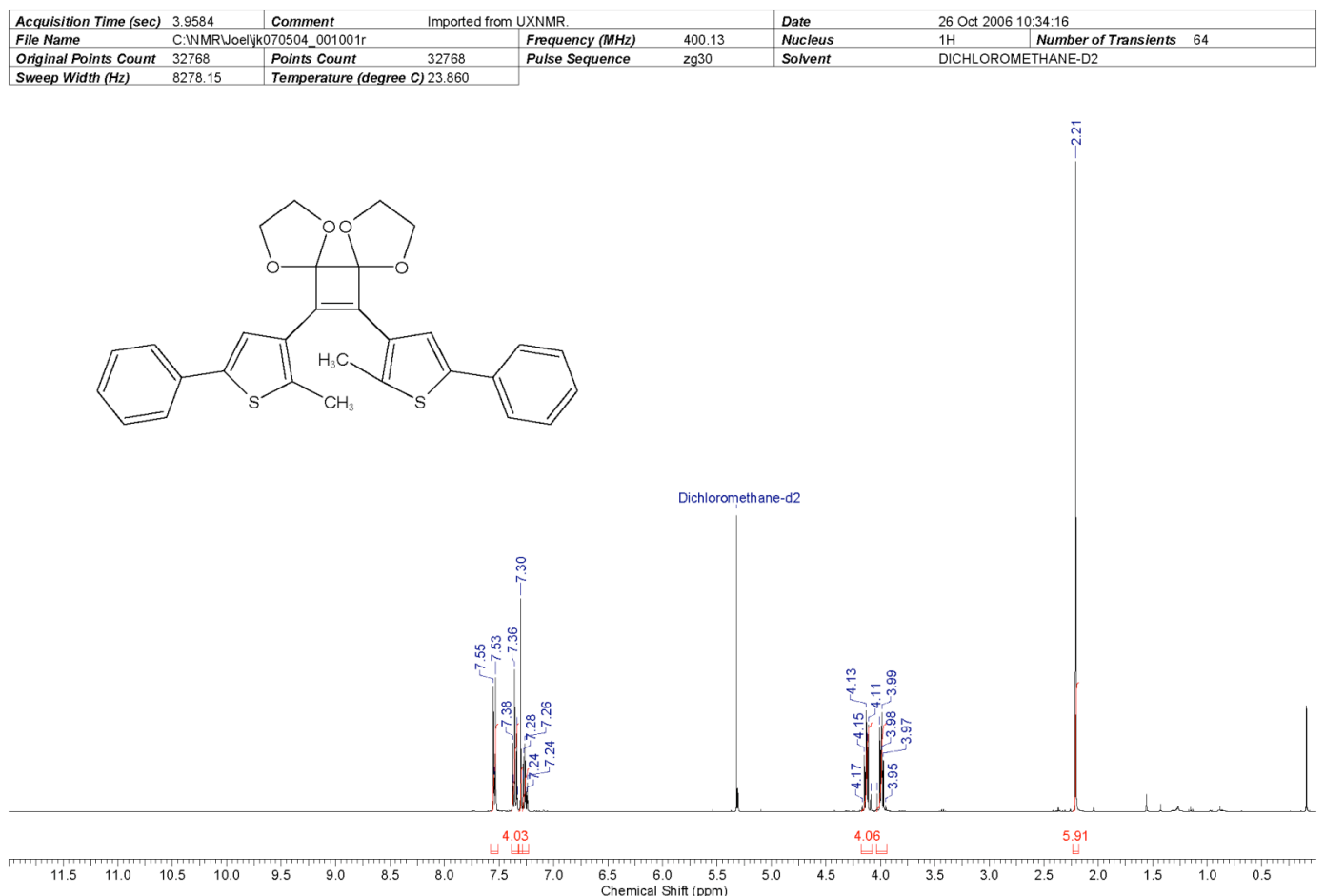

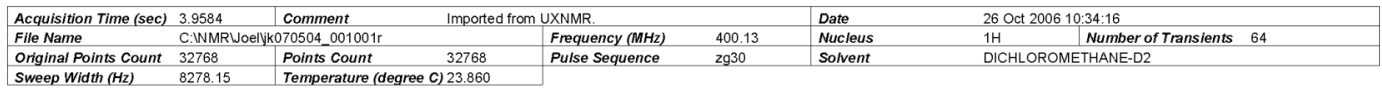

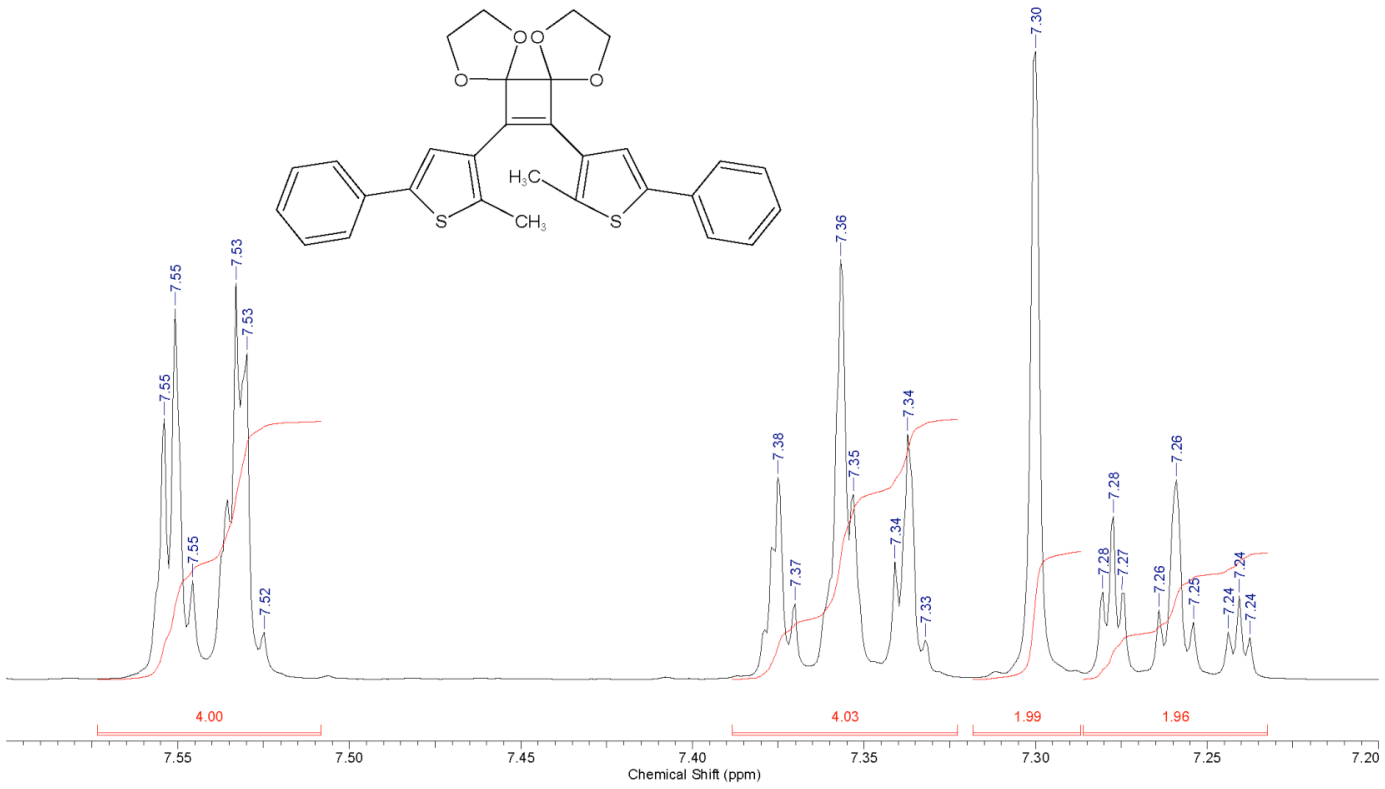



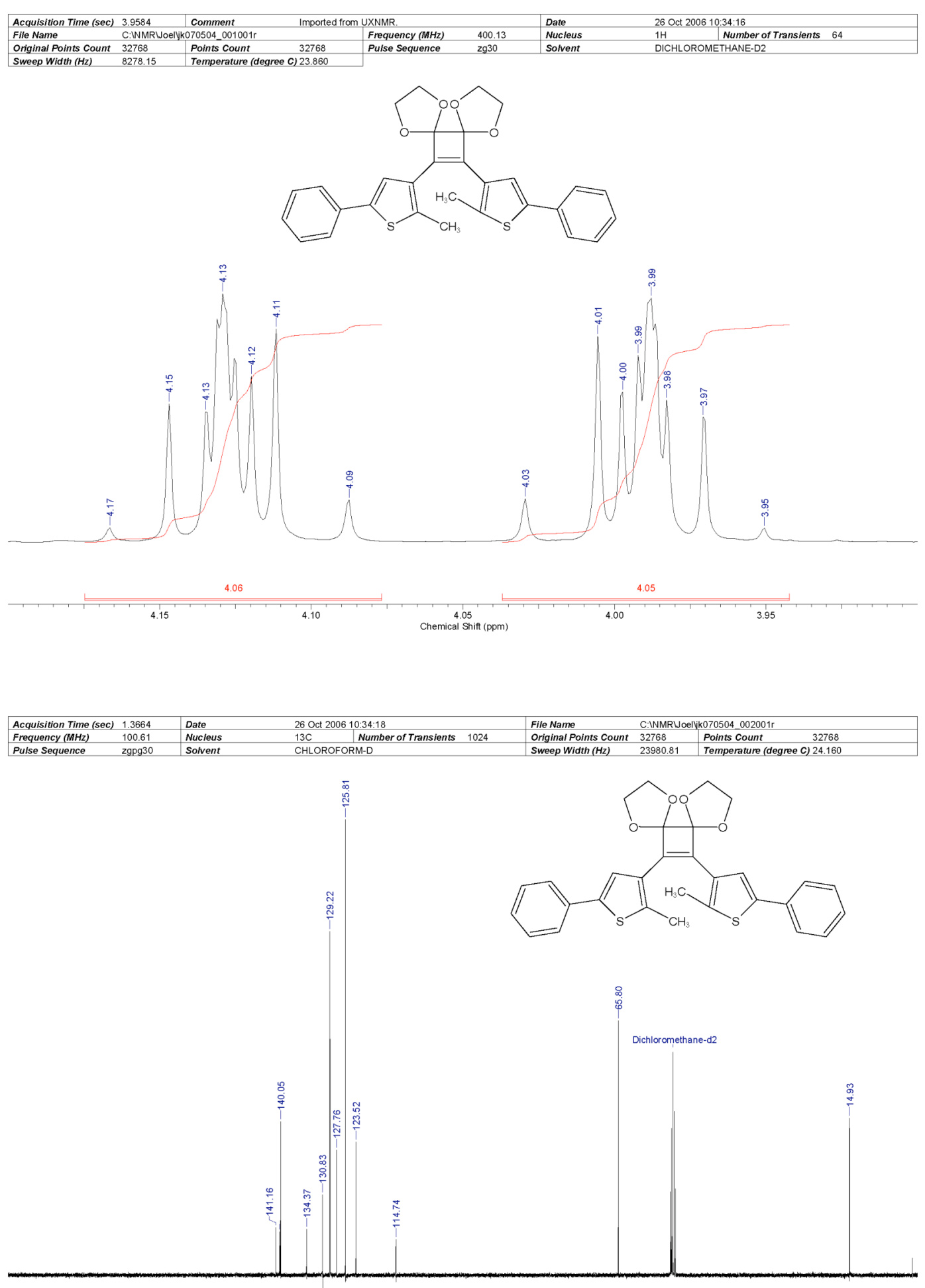

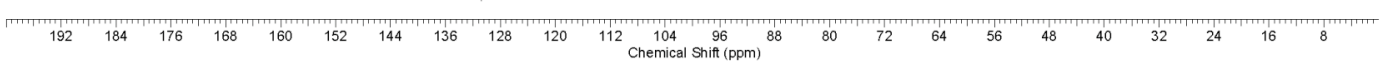




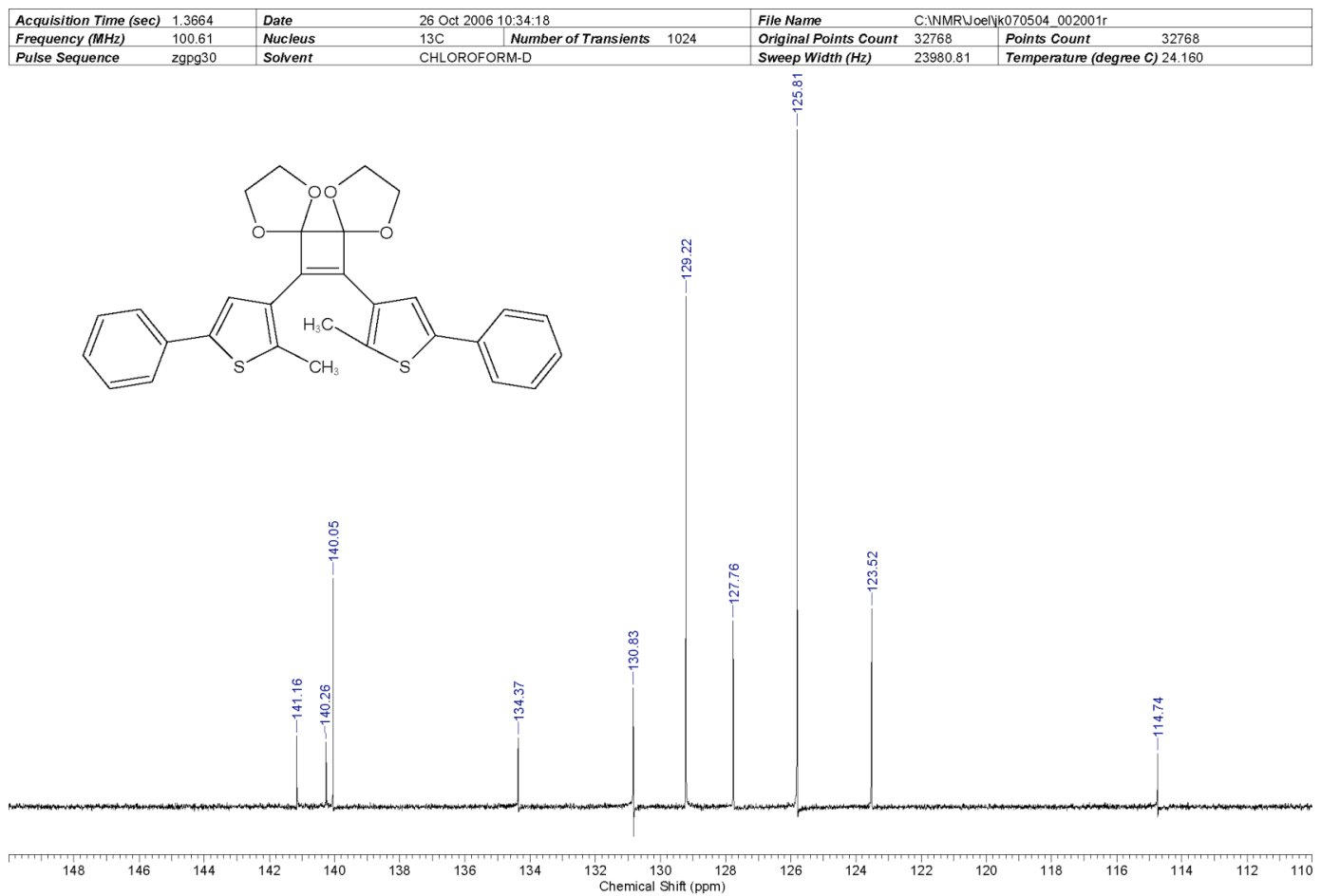




\section{Dimethyl 2,3-(2-methyl-5-phenylthien-3-yl)-1,4-butanedioate(5)}

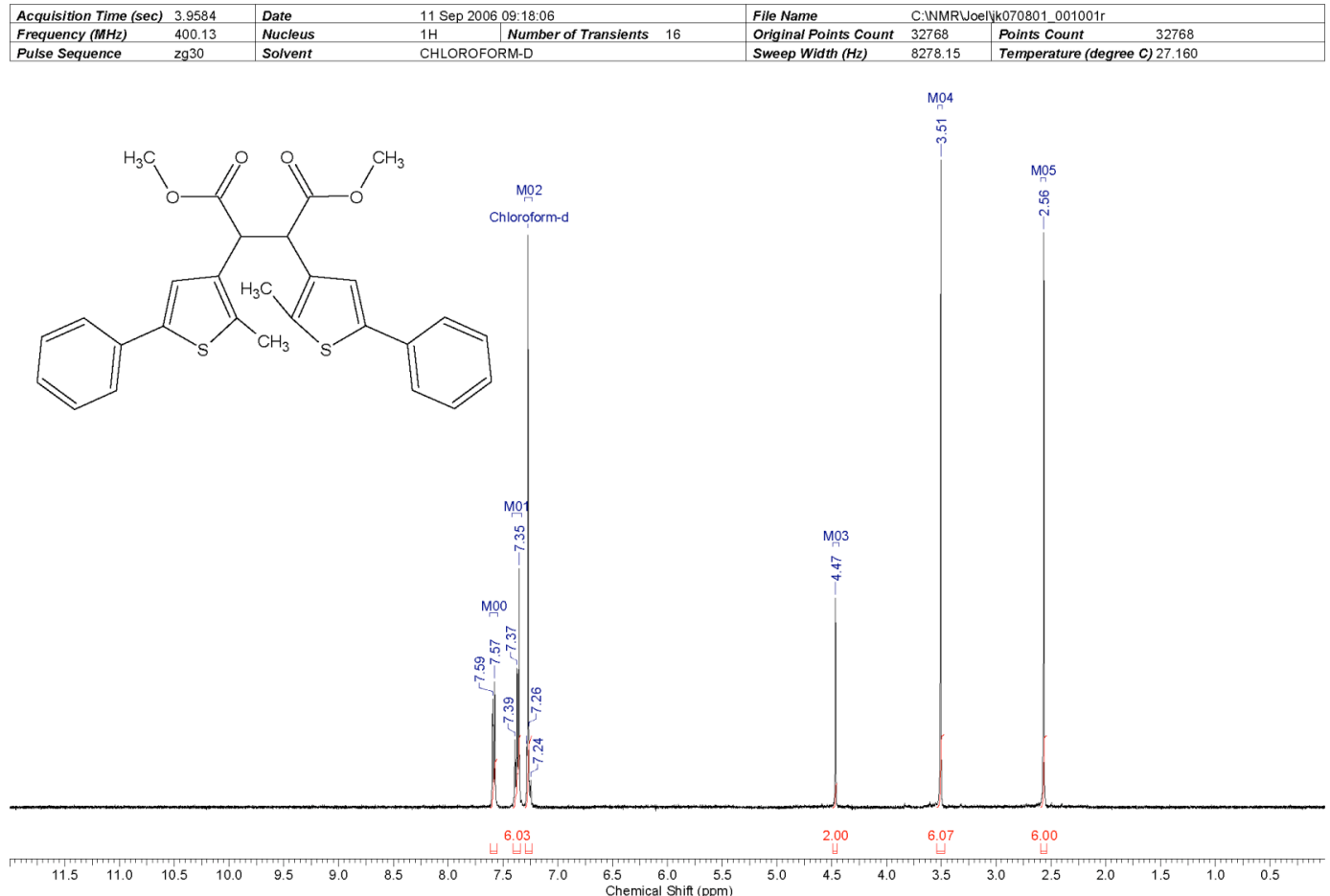

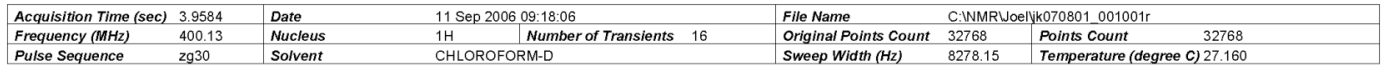

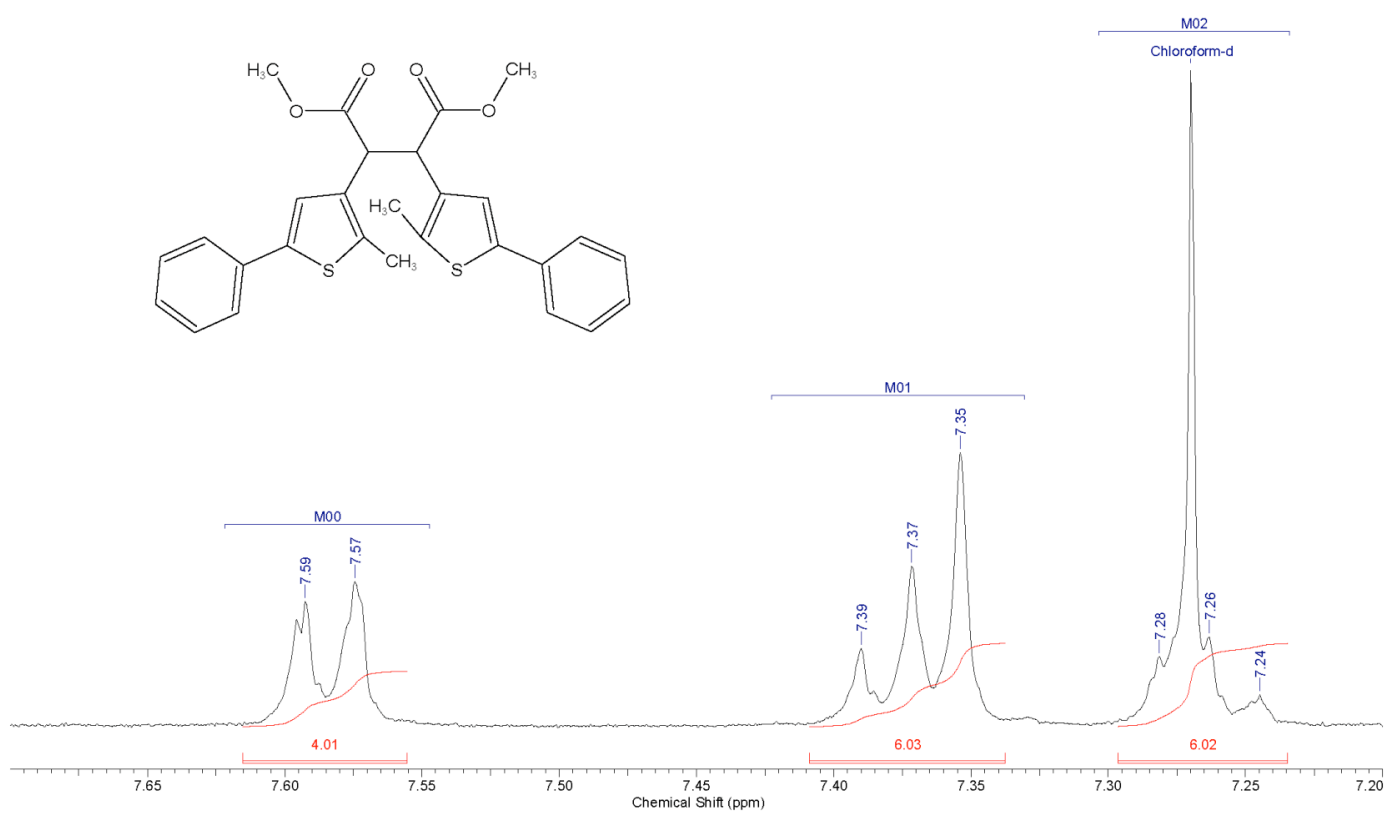




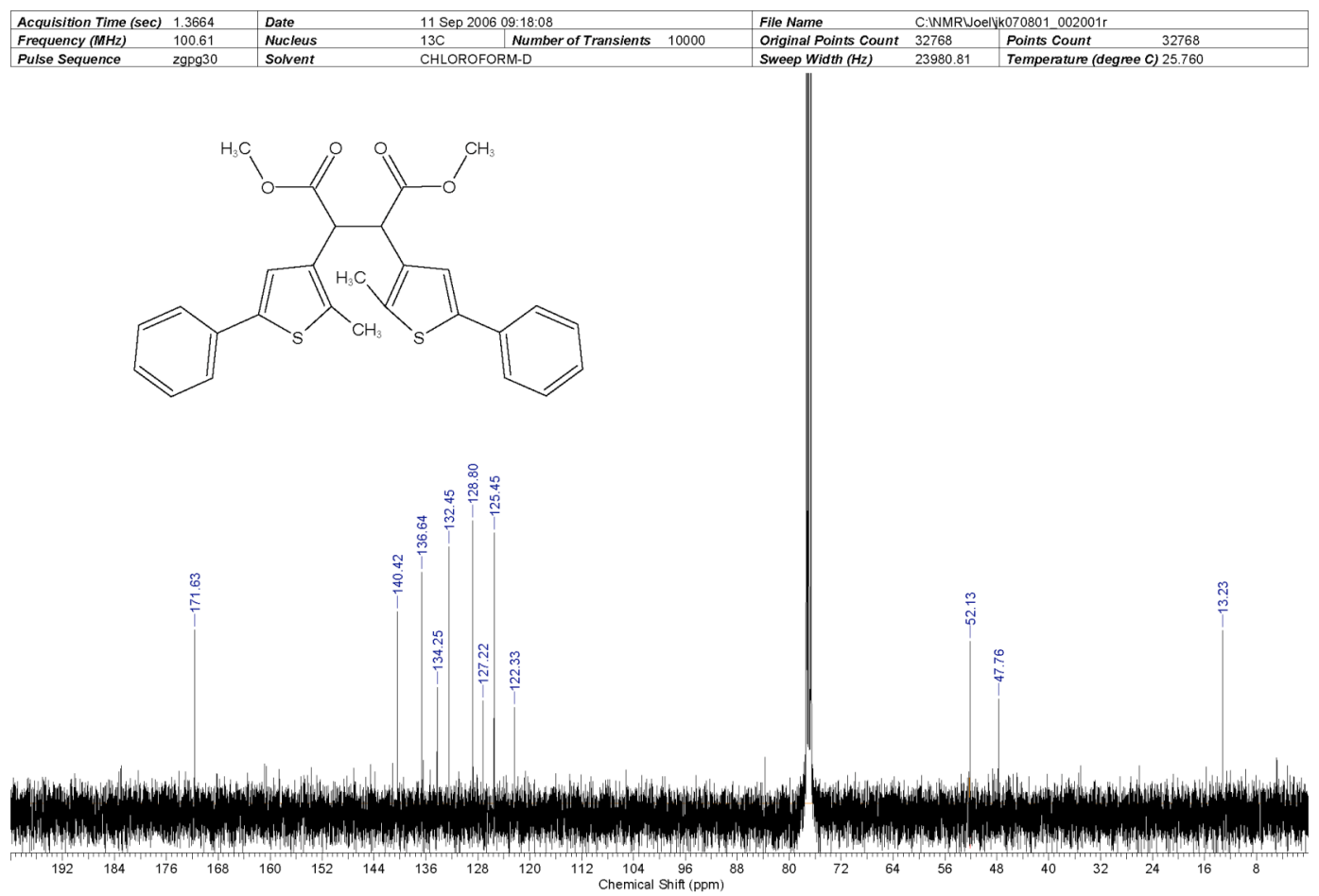

\section{Reference}

S1 Irie, M.; Lifka, T.; Kobatake, S.; Kato, N., J. Am. Chem. Soc., 2000, 122, 4871. 